\title{
Elaborating Research Questions Along The Writing-as-Inquiry Model
}

\author{
Jeremías P. Contell \\ University of the Basque Country (UPV/EHU) \\ jeremias.perez@ehu.eus
}

\author{
Oscar Díaz \\ University of the Basque Country (UPV/EHU) \\ oscar.diaz@ehu.eus
}

\begin{abstract}
Research Questions (RQs) drive, frame and shape research endeavours. Though classification schemas are available, it is not clear how RQs are developed. This work looks into the writing-as-inquiry model. According to this model, writing unleashes mental processes that help to further refine the discourse. Hence, we consider writing not for dissemination purposes but as an enabler of $R Q$ elaboration. This model fits the gradual and iterative process of $R Q$ development by iterating along two workspaces: the Content workspace, for idea profiling, and the Rhetorical workspace, for narrative construction. Unfortunately, current editors fall short to support this process. This work introduces the notion of "round-trip editors" in an attempt to account for this two-workspace iteration. Abstracting from experiences on a proof-of-concept artefact (i.e. DScaffolding), we introduce some general requirements that are informed by two main kernel theories: the knowledge-transforming model of writing and the writing-as-inquiry theory. DScaffolding is formatively evaluated for its utility and usability in elaborating problem-solving RQs.
\end{abstract}

\section{Introduction}

A research question (RQ) is an answerable inquiry into a specific concern or issue [1]. Broadly, RQs play three key roles [2]. First, defining the research scope, i.e. reducing the research focus from broad statements to specific questions to answer. Second, guiding the research process, i.e. selecting the most adequate course of action that better suits the type of research question. And third, positioning the contributions, i.e. opposing your work against someone else's to underline the originality of the contribution. These key concerns vindicate the importance of coming up with good RQs, and hence, the importance of guidelines that help in this endeavour. Unfortunately, a recent survey concludes that "to the best of our knowledge, no such guidelines exist" [2]. Hereafter, Thuan et al. introduce an analytical framework to typify RQ categories. By providing the main constructs that conform each kind of $\mathrm{RQ}$, this typology is tremendously useful for researchers to select the kind of RQ to be tackled, and hence, improving focus and driving artefact design. Yet, novice researchers, particularly $\mathrm{PhD}$ students, might be presented with different RQ examples, yet struggling to come up with their own [3]. The issue might go beyond the RQ constructs themselves to the process of instantiating those RQ constructs.

We observe that understanding a RQ not only requires a succinct $R Q$ statement but a thrilling Introduction section that helps readers situate and contextualize the rationales that underpin the RQ. If this happens for RQ understanding, we can conjecture that coming up with a RQ implies a similar journey. Guidelines for writing the manuscript's Introduction emphasize the need to first outline the research framework (i.e. practice, problem, related work, kernel theories, etc.) that will help readers fully grasp the interest of the target $\mathrm{RQ}[4,5,6]$. In a similar vein, RQ elaboration might well involve a similar discerning pathway. We can then wonder whether a sort of introduction-writing effort might unleash the mindset that would end up in a succinct RQ. On these grounds, Introduction writing would become a means to deepen, contextualize and synthesize RQs. This insight aligns with previous works on the benefits brought by the interplay of research \& writing that lead to the writing-as-inquiry model [7, 8, 9]. Rapley outlines this insight: "Writing is thinking. It is natural to believe that you need to be clear in your mind what you are trying to express first before you can write it down. However, most of the time the opposite is true. You may think you have a clear idea, but it is only when you write it down that you can be certain that you do" [10]. Research and writing reinforce each other, turning this interplay into a vehicle to reflect and come up with new insights $[11,7]$. Our work explores writing as an enabler for RQ elaboration. 
We believe RQ elaboration to be specially suited to writing-as-inquiry. Rationales are manifold. First, coming with a RQ is being described as an explorative, drill-down endeavour that requires reflection and self-dialogue, i.e. inquiry. The nature of the task (i.e. RQ development) fits the tool (i.e. writing-as-inquiry) . Second, RQ development is limited in scope. Writing wise, setting the rationales for a RQ should take the length of a manuscript's Introduction. This limited scope facilitates focus and adoption w.r.t writing the whole manuscript. Third, RQs have been typified. Templates exists that help in driving the writing towards instantiating the template for the case at hand. This guided writing might certainly reduce the anxiety behind the blank page syndrome [12], overcoming the common resistance of students to write [9]. Fourth, the output of the process is not just the RQ, but also the writing that sustains that RQ. This writing effort is to be capitalized later on during the manuscript writing up. Hence, writing is not just a byproduct of RQ development but becomes a main output of the research effort. It is just a question of advancing that effort for the sake of RQ obtention.

Nevertheless, when it comes to student adoption, previous arguments might fall short. Writing-as-inquiry as a means to RQ elaboration might be jeopardized by students' apprehension to writing. More to the point, this writing is not a one-shot effort (as opposed to manuscript writing) but RQ elaboration is a continuous effort to be held throughout. Different reports observe that the fact that scientific writing skills will be useful later in life is not sufficient motivation for students to practise those skills [12]. We can anticipate similar results if writing-as-inquiry is not accompanied by appropriate tools that excel in usability and consumability (e.g. easy to install and operate). Most current editors (e.g. Word) are generally thought for the product of writing rather than for the process of writing. They care about the product (i.e. the writing) by providing formatting and spell checking. But they behave alike no matter the current process step, i.e., whether you are drafting the first version or embellishing the ultimate version. However, the knowledge-transforming model of writing posits that writing is conducted at two different mental spaces [11]. In the Content space, the issues about knowledge, problem analysis and hypotheses are considered. By contrast, the Rhetorical space tackles how to express the Content issues. Thereby, Bereiter's model puts the focus on the process of writing, and the interplay of two mental spaces (i.e. the Content space \& the Rhetorical space). Unfortunately, the diffusion of these theories is curtailed by the limited support given in current editors.
According to Bereiter's model, a process-minded editor should support not just the Rhetorical space (as traditional editors do) but also the Content space, and, what is most important, a seamless integration between both. Since this activity is conceived as going back and forth between these two spaces, we coin the term "round-trip editors". This leads to our RQ which is described along Wieringa's template for design problems [6]:

How to design round-trip editors

that satisfy usability and consumability

so that students can benefit from writing-as-inquiry for RQ elaboration

in their Design Science projects

The focus is on students and Design Science Research (DSR), though we see no impediment to generalize the insights to other stakeholders and research methologies. By addressing this RQ, we make three contributions. First, we introduce "a nascent Design Theory" to identify constructs of utility and efficacy to support round-trip editors (Section 3). Second, we flesh out this theory through DScaffolding, a round-trip editor for LaTeX (Section 4). Finally, we provide first evidences about the benefits of Bereiter's knowledge-transforming model for the elaboration of RQ based on a focus group evaluation (Section 5). We start by introducing the kernel theories.

\section{Scientific Writing Theories}

Next paragraphs introduce theories that characterize the act of writing as being (1) two-space round-trip process; and (2), reflective.

Writing as a two-space round-trip process: the knowledge-transforming model of writing [11]. Scientific writing comprises different activities from idea generation, audience consideration or narrative exposition. Bereiter et al.'s model posits that writing is conducted at two different mental spaces (see Fig. 1). In the Content space, the issues about knowledge, problem analysis and hypotheses are considered. By contrast, the Rhetorical space tackles how to communicate the Content issues. Work in the Content space will encompass reflection on the meaning of the data in terms of the investigation problem, while work in the Rhetorical space will encompass the communication of that meaning to the audience. The output from each space serves as input to the other, so that questions concerning language and syntax choice reshape the meaning of the Content, while efforts to express the Content direct the ongoing composition. It is this 


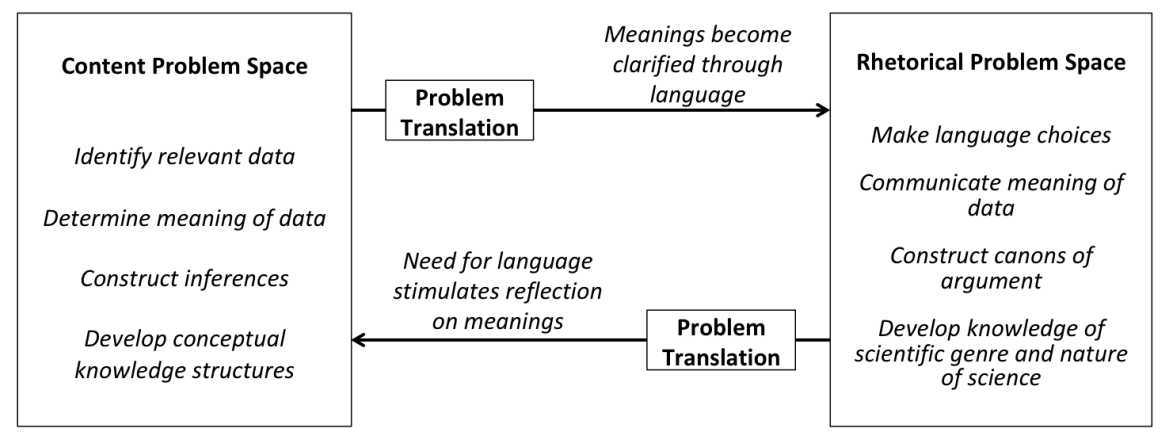

Figure 1. Bereiter and Scardamalia's knowledge-transforming model of composition (italics are taken from [13])

interaction between both spaces, according to Bereiter and Scardamalia, which provides the stimulus for reflection in writing. This lines up with the contenders of writing-as-inquiry.

Writing as a reflective process: the writing-as-inquiry model [7]. According to this theory, writing unleashes mental processes that help to further elaborate the discourse. Writing for an audience requires the writer to be sufficiently detailed and explicit to avoid ambiguity and misinterpretations [14]. As a result of this effort, new doubts and insights might arise that help to enhance the discourse so far. In the same vein, Wellington states "writing should be seen as knowledge developing rather than knowledge telling. This implies that writing should start on day one of the post-graduate 'journey' and is a means to develop thinking and understanding - as opposed to a process which simply transfers thoughts from brain to paper" [9].

\section{Operationalizing the theory: round-trip editors}

This section introduces a set of generalized requirements for round-trip editors (see Table 1). They are informed by the characteristics of the practice (i.e. Design Science Research (DSR) as the research methodology [5]), the problem (i.e. belated writing), the stakeholders (i.e. PhD students), and existing kernel theories (i.e. the writing-as-inquiry theory).

Provide support for the co-existence of two distinct workspaces: the Rhetorical workspace and the Content workspace. Kernel theories posit that writers might come up with new ideas while elaborating on the narrative. Authors depict this scenario as two different mental spaces, i.e. the Content space vs. the Rhetorical space (see Fig. 1). The question arises about whether these different mental spaces should also be realized as two different workspaces. We believe so based on the specifics of scientific writing: gradual elaboration and revision driven.

Gradual elaboration. DSR implies gradual and iteratively building up along three cycles: the relevance cycle, the rigor cycle and the design cycle [15]. If we accept the notion of writing as a form of inquiry about relevance, rigor and design, it just follows that writing should also be conducted in the same way as research: gradually. So while we are often accustomed to writing up at the end of our research process, the writing-as-inquiry theory advocates for writing to be initiated as soon as possible, not just for documenting purposes, but for research purposes!

Revision driven. Peer revision is another research practice [16]. However, not all reviewers are the same. On one side, experts (e.g. supervisors) can give feedback on Content. On the other side, for non-experts the best feedback that can be gotten from them is not on Content, it is on clarity, i.e. the Rhetorical space. This input should not be undervalued. Lack of clarity is a too common complaint on manuscript revision [17]. Therefore, these two kinds of reviewers (experts vs. non-experts) complement each other but each has different communication needs. Supervisors know about the problem. Their interest is primarily on the rationales and contributions, to be novel and well argued. This can be better captured at the Content level where the analysis is cleansed of all "the Rhetorical trim". By contrast, non-experts care about the narrative flow and clarity, i.e. they work at the Rhetorical level. Therefore, if writing starts from the outset, both realms should be kept throughout the research process so that the appropriate level of communication is kept for each kind of interlocutor. Therefore, round-trip editors should support the co-existence of two distinct workspaces throughout time.

Provide a head start on the Rhetorical workspace. Round-trip editors should give writers a head start by automatically generating a first textual draft out of the Content space. Rationales are twofold. First, to fight back the blank page syndrome. During writing, different 
Table 1. Meta-requirements for round-trip editors.

\begin{tabular}{|l|l|l|}
\hline Meta-requirement: Provide support for ... & Source & Argumentation \\
\hline ...the co-existence of two distinct workspaces & $\begin{array}{l}\text { Process } \\
\text { characterization }\end{array}$ & $\begin{array}{l}\text { The writing-as-inquiry theory: } \\
\text { elaboration \& Revision-based }\end{array}$ \\
\hline ...a head start on the Rhetorical domain & $\begin{array}{l}\text { Stakeholder } \\
\text { characterization }\end{array}$ & $\begin{array}{l}\text { Prone to the blank page syndrome \& } \\
\text { Unawareness of structuring conventions }\end{array}$ \\
\hline ...keeping both workspaces in synchrony & Kernel Theory & The knowledge-transforming model of writing \\
\hline ...quality checking in the Content realm & $\begin{array}{l}\text { Stakeholder } \\
\text { characterization }\end{array}$ & $\begin{array}{l}\text { Hesitant on when to start writing \& Hesitant on } \\
\text { how much reading or RCA is enough }\end{array}$ \\
\hline
\end{tabular}

concerns should be considered: adjusting to audience, sequencing how content issues are ordered; backing statements with suitable references; usage of appropriate connectors for smooth reading. This plethora of concerns causes a kind of paralysis that frequently leads to procrastinating writing [18].

But even in the absence of the blank page syndrome, writing efforts might be diluted due to unawareness of the conventions for paper structuring. Similar insights are stated in [19]: "it is useful to guide novice researchers in writing their research introductions more systematically and effectively, especially with appropriate use of transitional words and phrases". This calls for students not being left alone in transitioning from the Content space to the Rhetorical space. Rather, a first draft can be generated out of the Content space that includes a tentative structure, connectors and references. This draft acts as a kind of textual template where students need to work out the narrative, but "the ingredients" are already there. Note that this draft should be completely subject to change, with the sole objective of providing students with a starting point.

Provide support for keeping the Rhetorical workspace and the Content workspace in synchrony. First meta-requirement advocates for the existence of two separate workspaces. These workspaces reflect the same research instance but stress different concerns: argumentative vs. communicative. But these concerns influence each other. The writing-as-inquiry theory posits that the act of writing causes writers to reflect, to alter and to reconsider what they had in mind before they write it up. New causes of the problem, causal relationships, concepts or insights might pop up during the writing (a.k.a. light bulb moments). While communicative efforts will have no impact, the emergence of new ideas while writing should find a counterpart in the Content realm. And vice versa, upgrades of the Content workspaces need to be propagated to the Rhetorical workspace (e.g. adding some boilerplate paragraph in the text). Students should be able to move between workspaces at wish depending on their current interests, and let editors keep them in sync.

Provide support for quality checking in the Content workspace. Students commonly wonder about whether they have read enough or if the number of causes/consequences identified is sufficient to sustain the importance of the problem. These questions are related with the quality of the problem analysis being conducted. In the same way that spell checkers validate the Rhetorical workspace for conformance along grammatical rules, "RCA checkers" might be envisaged to assess the quality of the Content workspaces. As a case in point, Baer et al. propose comprehensiveness as a primary metric to judge the success of the problem formulation activity [20]. Comprehensiveness is defined as "the extent to which alternative, relevant problem formulations are identified with respect to an initial symptom or web of symptoms [...] A set of formulations that addresses only a subset of symptoms is hence considered to be less relevant and, as a result, less comprehensive than a set that addresses the entire web of symptoms [...] without considering irrelevant symptoms" [20]. Metrics can then be elaborated to assist students to assess the comprehensiveness of their RCA.

Quality checking might also help to determine when to kick off the Rhetorical endeavor. Indeed, Wellington's students express the distress of not knowing when to stop reading and start writing [9]. Supervisors can set some criteria for good-enough RCA that help students to move on to the next stage rather than keep looking for e.g., additional references in the literature. Procrastination can take many forms, and RCA is not free from "polishing the round ball". This does not mean that RCA has a set end. Not in DSR where the relevance cycle calls for researchers to keep looking for new evidences throughout the project. The good-enough gauge would indicate that sufficient insights have been collected to start the writing up, but not that RCA ends.

These (meta) requirements conform a "nascent Design Theory" to identify constructs of utility and efficacy to support round-trip editors. But this theory needs to be validated: do round-trip editors really 
promote early writing? Does the separation of spaces facilitate revision or rather implies an additional cognitive burden? To what extent does the head start lessen the blank page syndrome? Checking this out requires these meta-requirements to be fleshed out in a "purposeful artefact".

\section{Realizing the meta-requirements: DScaffolding}

Previous meta-requirements do not preclude the architectural solution of round-trip editors. Nevertheless, three architectures can be envisaged for this artefact. First, a Rhetorical-first architecture that departs from existing word processors. Here, a text editor is enlarged with "conceptual utilities" that help capture notions and relationships of the domain at hand. Second, a Content-first architecture that enlarges existing conceptual-map editors with text generators that derive boilerplate text out of the maps. Finally, a mediator approach that rests upon drivers for existing editors to interplay. Here, keeping the Content realm (in editor A) and the Rhetorical realm (in editor B) in sync is realized through API calling between editor A and editor B. Our artefact, DScaffolding, follows a mediator architecture whereby two existing editors are coordinated: i.e. MindMeister ${ }^{1}$ and Overleaf ${ }^{2}$, that account for the Content workspace and the Rhetorical workspace, respectively

DScaffolding is fully functional and available for download at the Chrome's Web Store ${ }^{3}$. Next, we delve into how DScaffolding realizes the aforementioned requirements.

\subsection{Provide support for the co-existence of two distinct workspaces}

DScaffolding resorts to mind maps and LaTeX as the formats for the Content realm and the Rhetorical realm, respectively. LaTeX is a markup language widely used in academia for scientific writing. As for mind maps, evidences have been reported about the use of maps for scaffolding the writing process that "facilitates an improvement in the expression of scientific knowledge and concept understanding in pupils writing" [21].

In mind maps, ideas are radially disposed around a root node. This structure can be pre-set through a map template, i.e. a set of labeled nodes that can be later expanded by adding new child nodes. For DSR, this template serves to guide students about the different concerns raised during RQ elaboration.

\footnotetext{
${ }^{1}$ https://www.mindmeister.com/

${ }^{2}$ https://www.overleaf.com

${ }^{3}$ https://rebrand.ly/DScaffolding-extension
}

This template very much depends on the RQ type. Thuan et al. identifies three types of RQ in DSR: problem solving, gap spotting, and problematization [2]. The former is by far the most popular, accounting for $77 \%$ of the papers being analyzed [2]. We conjecture problem-solving to be even more popular among $\mathrm{PhD}$ students whose youth makes them nominally more conversant with technological solutions that conscious about new domain problems. In addition, problem-solving RQs might specially benefit due to their development involving the combination of exploration (i.e. finding/elaborating the problem) and building of purposeful artefacts. We then focus on problem solving.

Wieringa introduces a template for Design Problems [6] (see this paper's introduction for an example). Therefore, it could be said that the Introduction should provide enough hints for readers to assess the distinct elements of Wieringa's template. The template is not enough but needed is assistance on coming up with the storyline that underpins the RQ. To this end, we capture the distinct elements of Wieringa's template in terms of a mind map. Fig. 2 shows such a map for this very paper. Researches can document their RCA progress through this map, adding, removing, gathering nodes at wish, using MindMeister facilities. From this perspective, DScaffolding turns MindMeister into a RQ-dedicated editor.

\subsection{Provide support for quality checking in the Content workspace}

RCA goodness criteria (e.g. comprehensiveness [20] (see Section 3)) might be realized as heuristics to assess whether the RCA conducted so far is good enough. Specifically, two heuristics can be considered:

- the larger the RCA structure, the more elaborated the analysis. Some thresholds can be set in terms of the number of consequences/causes being analyzed as well as the depth to the causal tree.

- RCA strength is not only based on its causal depth but also on the quantity and quality of the supporting evidences. An evidence threshold can be set as the total count of leaf nodes that point to literature references or data analysis.

These heuristics can be operationalized through thresholds set by the students' supervisors through DScaffolding's configuration page. For instance, supervisors can set a given number of consequences/causes students need to come up with before moving to the Rhetorical workspace, and in this way, help student assess when they have read enough. 


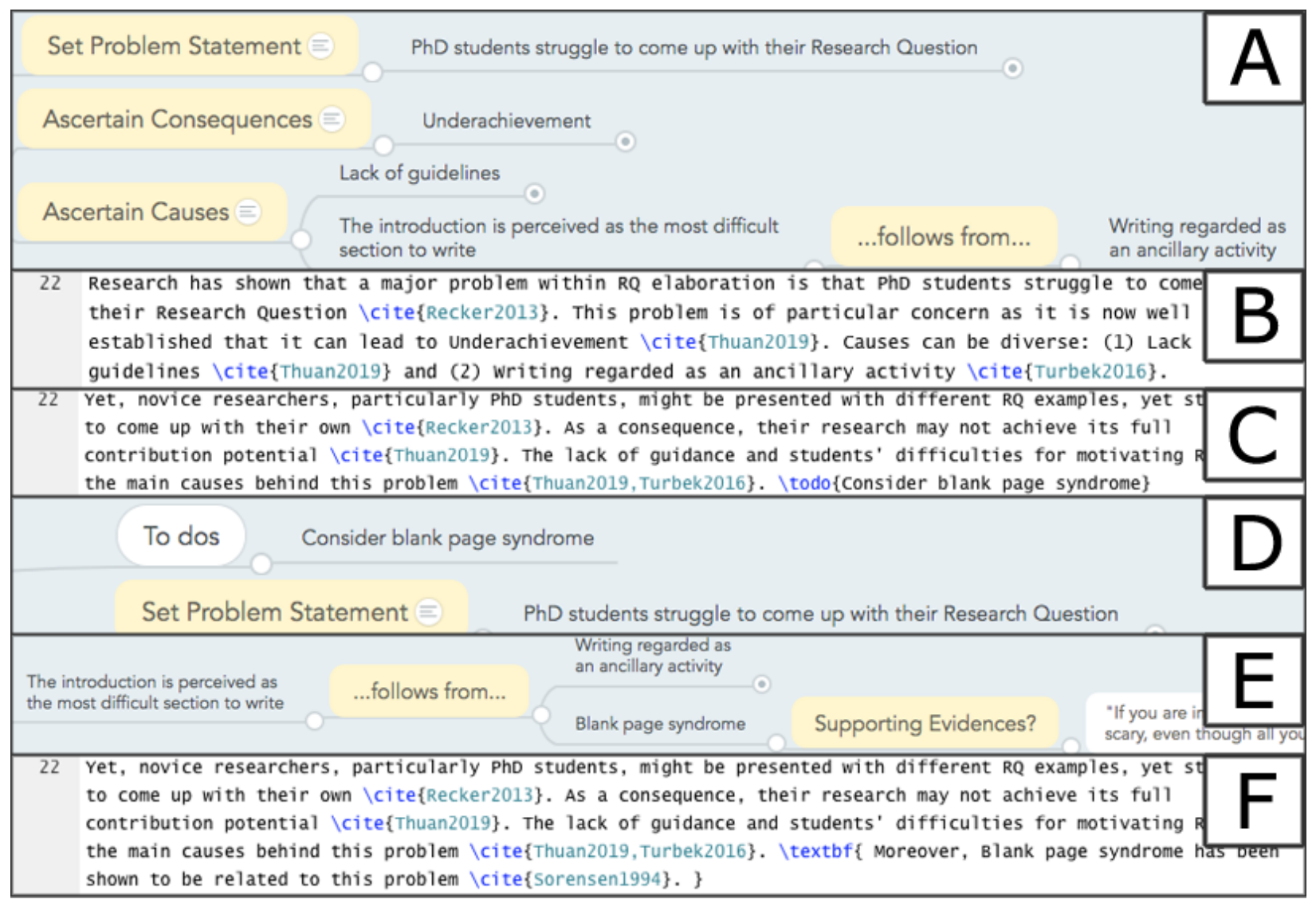

Figure 2. Synchrony between the Content and Rhetorical workspaces. See full map ${ }^{4}$ and video of the process ${ }^{5}$.

\subsection{Provide a head start on the Rhetorical workspace}

Once the Content model is sufficiently complete, students can move to the Rhetorical workspace, i.e. LaTeX editors. To combat the blank page syndrome, DScaffolding creates a first LaTeX draft out of the current Content model. To this end, DScaffolding resorts to model-to-text transformations (not addressed here). Broadly, some connectors and boilerplate paragraphs cook up a reasonable starting point for students to elaborate on the narrative. Good sequencing practices from [5] are wired-in, whereas connectors are taken from those suggested in [22]. Fig. 2 shows the output for the running example. Bibliographical references (i.e. the bibtex file) are also obtained from the evidences collected in the mind map. From now on, students can elaborate on this raw draft.

\footnotetext{
${ }^{4}$ https://rebrand.ly/StrategicWriting-mindmap

${ }^{5}$ https://rebrand.ly/StrategicWriting-video
}

\subsection{Provide support for light-bulb moments while in the Rhetorical workspace}

The writing-as-inquiry theory predicts that new insights come up during writing. This kind of lateral thinking is not exclusive of writing. It happens in other domains. Specifically, DScaffolding gets inspiration from Integrated Development Environments (IDEs) in Software Engineering. Here, new concerns might also arise when programming. To prevent lost focus, IDEs provide so-called "task tags". For instance, Eclipse realizes task tags as comments programmers inlay into code, used as reminders of actions, work to do or any other action required by the programmer. These tags might be interpreted by the IDE. For instance, the tag "//TODO class to be revised" will cause blue mark on the right-hand gutter so that programmers can jump to all points in the current code file where work is needed [23]. Likewise, DScaffolding introduces a LaTeX command, i.e. " $\backslash$ todo possible cause could be ...\}". This command is transparent to LaTeX but not to the model transformer. When back to the Content realm (i.e. MindMeister), the mind map is refreshed with a 
new todo node.

\subsection{Provide support for keeping the Rhetorical workspace and the Content workspace in synchrony}

Students move between workspaces at wish. They can opt to further elaborate the RCA (e.g. adding new consequences or reading for evidence searching) or instead, strive to better communicate the ideas elaborated so far (e.g. rephrasing sentences, removing paragraphs). The challenge: transparently conducting counterpart actions so that both workspaces are kept in sync. Change propagations might be needed in both senses, i.e. from MindMeister (i.e. the Content workspace) to $\mathrm{LaTeX}$ (i.e. the Rhetorical workspace), and vice versa. This is realized through model-to-text transformations (not addressed here).

As an example, consider the Content space in Fig. 2(a). Right-click for DScaffolding to generate some boilerplate sentences that verbalize this content (Fig. 2(b). Now, students can re-phrase this text through their own words. In the process, they might come up with new insighst about possible causes that they might have heard about in the past but now emerges, e.g., the blank-page syndrome. To avoid losing focus while writing, they just write down the insight by typing "\todo\{consider blank page syndrome\}", and continue writing (see Fig. 2(c)). At some point, they move back to the Content Realm, i.e. the mind-map is re-opened. On loading, DScaffolding locates $\backslash$ todo \{\} in the Latex counterpart, and shows them up in the mind map (see Fig. 2(d)). Students can next look for evidence about the blank page syndrome. On their way, students might come across with additional quotes about the importance of the problem, and accordingly enrich the map (see Fig. 2(e)). Eventually, students go back to the Rhetorical realm, i.e. LaTeX (see Fig. 2(f)). Notice that this does not result in loss of user-edited text. Rather, previous text is now enriched with some boilerplate text about the recently added map nodes (i.e. blank-page syndrome). Worth noticing new paragraphs are not added at the document's end but inlayed near related paragraphs (e.g. paragraphs describing other causes). To this end, DScaffolding resorts to approximate string-matching techniques [24]. Bold font is used to easily identify newly injected fragments within existing text.

\section{Evaluation}

This evaluation aims to inform whether $D$ Scaffolding is an appropriate conduit for writing-as-inquiry. The argument goes as follows: if students appreciate the benefits of writing-as-inquiry, then they will embrace these benefits at the moment when they are most needed, i.e. at the project onset. Nevertheless, we do not evaluate here the validity of this causal relationship, i.e. benefit awareness leads to early writing adoption habits. We need first to test out that DScaffolding is a suitable means for writing-as-inquiry. To this end, we conducted a focus group evaluation. A focus group is defined as "a moderated discussion among 6-12 people who discuss a topic under the direction of a moderator" [25]. We conducted formative evaluation (i.e. getting prompt feedback about the artefact design) w.r.t. two main adoption criteria: usability and effectiveness. The focus was particularly on (1) assessing the extent moving back-and-forth between the two workspaces was conducted with ease, and (2), the benefits derived from writing.

Identify Sample Frame. Focus group wise, Morgan suggests a lower boundary of 4 participants and an upper boundary of 12 participants [26]. Participants should be representative of potential stakeholders. To this end, main selection criterion was the $\mathrm{PhD}$ life-cycle: if too preliminary, then research was too preparatory to make worth elaborating a first draft; if too mature, then the research was almost accomplished, reducing the chances of (and motivation for) light bulb moments. Four PhD projects qualified. This corresponds to post-graduates in their second year. Students were performant at using both LaTeX and MindMeister. English qualifications were $\mathrm{B} 2 / \mathrm{B} 1$, and previous writing experience was poor, mainly limited to writing their capstone projects. Publications were a must to successfully end their PhDs.

Conduct the Focus Group. Students were first introduced to the virtues of writing-as-inquiry. Next, DScaffolding was presented. After using DScaffolding at their own pace for two months, a focus group was conducted. The session lasted 2 hours in which the co-authors of this paper shared moderation and note-taking tasks. The discussion revolved around their experience using the tool. The session was then transcribed.

Usage. The participants stated that they had used DScaffolding for an average of 34 hours for a two-month period. This use was concentrated in the mind map in a ratio of 70 per 30 in the LaTeX realm. All the participants reported having completed at least four round-trips (understanding a round-trip as a mind map-LaTeX-mind map iteration). This number of iterations suggests the appropriateness of the co-existence of the two workspaces where activities in both realms intertwine throughout.

Generation of a head start. All participants appreciated the starting point generated by DScaffolding. One participant pointed out that it 
Table 2. Awakening Scenarios

\begin{tabular}{|l|l|l|l|l|}
\hline Category Subject & S1 & S2 & S3 & S4 \\
\hline Glossary & & I & I & \\
\hline Practice scope & I & & I & I \\
\hline Evidence shortage & I & I & I & I \\
\hline Related work & & I & & I \\
\hline Problem importance & & & I & \\
\hline
\end{tabular}

had impacted positively his confidence, as he had "been able to use it as a reference of good practices". Two participants found useful the inclusion of lexical bundles as a way to learn expressions that they "would not use otherwise". They stated that the amount of content that flowed from the mind map to LaTeX was appropriate, considering that "it can always be removed afterwards". As for the structure of the text, none of them altered the structure suggested by the head start template. Conversely, shifts in the default connectors were reported.

Usability. We resorted to the System Usability Scale (SUS) SUS yields an overall usability score in a 0-100 range, and in general, 70 is considered to be the threshold between good and marginal results. The calculated score for DScaffolding was 83 (see Fig. 3).

Effectiveness. Thematic analysis was conducted that ended up in five main codes for "awakening scenarios" (see Table 2): glossary (i.e. realizing used terminology was ambiguous), practice scope (i.e. unclear target audience or overclaims about the recipient practice), evidence shortage (i.e. dubious or "gut feeling" evidence), related word (i.e. poor, unframed comparison with related work) and problem importance (i.e. limited evidence of the importance of the problem). To our surprise, no student reports on coming up with additional causal relationships.

Threats to validity. Construct validity is especially challenging here due to the different factors that might influence the phenomena, and to which the researchers might not be aware of. At this respect, it is important to note that we are not evaluating DScaffolding's adoption but the appropriateness of DScaffolding to support writing-as-inquiry. Hence, factors such as curiosity about the tool or relationship with the tool creators that might blur the evaluation, do no apply here where students were "forced" to use DScaffolding. On the other hand, we can plausibly argue that the effectiveness of writing-as-inquiry for providing valuable input to the research process is correlated with the stage of the $\mathrm{PhD}$ life-cycle. We attempted to control this variable by selecting all participants being in their second year. We also suffer from external validity, i.e. the lack of generality. To fight this back, we paid special attention to profile the students along those factors that might impact writing. The research area, the level of pressure to publish, English skills or previous writing experience, are all variables that might impact this phenomena. By expliciting this setting, we hope this experience to be replicable. Finally, external validity looks at the generalizability of the results. Our research is motivated by limitations exposed for RQ in DSR after the work of Thuan et al. [2]. We can plausibly argue that round-trip editors might be useful to stakeholders other than students, and research methodologies other than DSR. But we do not have any evidence yet.

\section{Related Work}

This work proposes a treatment (i.e. writing-as-inquiry) to tackle a problem (i.e. struggling to come up with RQs) for a target population (i.e. novice researchers) in a given practice (i.e. Design Science Research). We claim no novelty on the use of writing-as-inquiry. Our contribution is on operationalizing this theory. Thereby, this work should be compared to efforts on operationalizing writing-as-inquiry in different settings and for distinct audiences. Table 3 frames our efforts within this movement along four main dimensions: population (i.e. target audience, target object), intervention (i.e. artefact being proposed), focus (i.e. whether the stress is on the product of writing or the process of writing) and outcome (i.e. the pursued aim).

Awareness of discourse conventions. Aim: helping novices familiarize with scientific writing. For the Creating a Research Space (C.A.R.S.) model, Mover is a tool that automatically breaks down introductions by mapping each fragment to one of the CARS elements [30]. Also for the CARS model, Research Writing Tutor (RWT) houses a database of exemplary scientific writing for each of the CARS steps [31]. At a finer grain, AWSuM autocompletes user input with the so-called "lexical bundles" extracted from the literature, putting examples of actual use [29]. Main difference with our approach is that these tools focus on the product, whereas round-trip editors move to the forefront the writing process: the need for explicitly supporting not only the Rhetorical realm but also the Content realm. And most importantly, the seamless transition between these two workspaces.

Fostering student writing. Aim: promoting early writing. First strategy, quick feedback. Research Writing Tutor (RWT) analyzes students writing pieces and automatically generates discipline-specific feedback [31]. SWoRD [28], in turn, is a web-based system 


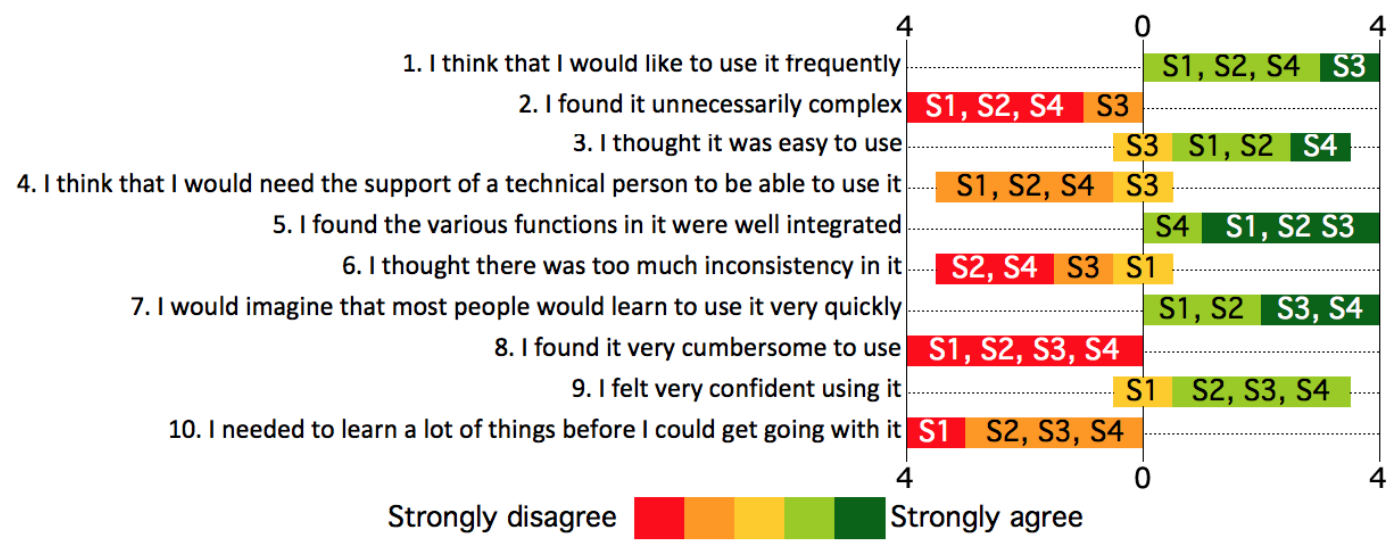

Figure 3. Diverging Stacked Bar Chart for the SUS Questionnaire using Likert Scales.

Table 3. Comparison of related work.

\begin{tabular}{|l|l|l|l|l|l|l|}
\hline Paper & Target audience & Target object & Intervention & Focus & Outcome \\
\hline $\begin{array}{l}\text { Research } \\
\text { diary [27] }\end{array}$ & $\begin{array}{l}\text { novice } \\
\text { researchers }\end{array}$ & insights & research diary & $\begin{array}{l}\text { writing } \\
\text { process }\end{array}$ & emotional support \\
\hline $\begin{array}{l}\text { SWoRD } \\
{[28]}\end{array}$ & undergraduates & paper & $\begin{array}{l}\text { simulate the journal } \\
\text { publication process }\end{array}$ & $\begin{array}{l}\text { writing } \\
\text { process }\end{array}$ & $\begin{array}{l}\text { improve writing \& reviewing } \\
\text { skills, gain content knowledge }\end{array}$ \\
\hline $\begin{array}{l}\text { AWSuM } \\
{[29]}\end{array}$ & $\begin{array}{l}\text { novice \& L2 } \\
\text { researchers }\end{array}$ & paper & $\begin{array}{l}\text { lexical bundle } \\
\text { autocompletion }\end{array}$ & $\begin{array}{l}\text { writing } \\
\text { product }\end{array}$ & $\begin{array}{l}\text { greater confidence \& } \\
\text { autonomy }\end{array}$ & and \\
\hline Mover [30] & $\begin{array}{l}\text { novice writers \& } \\
\text { L2 researchers \& } \\
\text { students }\end{array}$ & abstract & $\begin{array}{l}\text { article structure } \\
\text { analysis tool }\end{array}$ & $\begin{array}{l}\text { writing } \\
\text { product }\end{array}$ & discourse awareness & \\
\hline RWT [31] & $\begin{array}{l}\text { students } \\
\text { DScaffolding }\end{array}$ & $\begin{array}{l}\text { novice } \\
\text { researchers }\end{array}$ & Raper & $\begin{array}{l}\text { automated discipline } \\
\text { specific feedback }\end{array}$ & $\begin{array}{l}\text { writing } \\
\text { product }\end{array}$ & $\begin{array}{l}\text { improve text quality \& } \\
\text { discourse awareness }\end{array}$ \\
\hline
\end{tabular}

that relies on peer feedback for practicing writing skills in a classroom setting. Second strategy, self-dialogue. Engin presents research diaries as scaffolding tools that support the construction of knowledge while writing [27]. In a similar vein, academic blogs are also proposed for advancing understanding by "connecting the dots" [32]. Likewise, DScaffolding also encourages quick feedback and self-dialogue. Unlike previous approaches, these strategies expand beyond the Rhetorical realm (i.e. the writing itself) to the Content realm (i.e. the map mapping). This further promotes feedback and self-dialogue since the Content realm might promote thinking in the large (easy to grasp the whole picture) as opposed to thinking in the small (specific text paragraphs). These strategies might however need to be tuned based on the target object, i.e. helping obtain a paper, an abstract, a RQ or just writing down research insights.

We then consider round-trip editors to make a distinctive contribution to the operationalization of the writing-as-inquiry model by supporting two differentiated yet interrelated workspaces.

\section{Conclusions}

We advocate for the use of early writing as a means to develop Research Questions. Informed by the writing-as-inquiry theory, we introduced a set of general requirements for round-trip editors. These requirements are fleshed out through a "purposeful artefact", i.e. DScaffolding. Evaluation is still formative. So far, evidences point towards (1) the head start having utility for fighting back the blank page syndrome, and (2) synchronized workspaces having utility for jumping between the Content workspace and the Rhetorical workspace. During the evaluation, writing-as-inquiry manifested itself through different insights students come up during RQ elaboration. These "awakening scenarios" refer to terminology profiling, problem scoping, frame-based comparison of related work, and evidence support. These scenarios have much to do with research topics being socio-technical. 
Socio-technical problems require patience and careful consideration of the practice and the problem, patience that students frequently lack, eager to move to the solution space and start coding. The question is when would have those "awakening states" come about, should not students being forced to use DScaffolding. In some cases, supervisors might spot these gaps. Far too frequently, conference reviewers are those pinpointing these concerns. But then, it is too late. Our hope is that the benefits suggested by the writing-as-inquiry theory will offer students a compelling motivation not just to write, but to write early on the effort to elaborate their research questions.

\section{Acknowledgements}

This work is co-supported by MCIU/AEI/FEDER, UE under contract RTI2018-099818-B-I00.

\section{References}

[1] D. Kowalczyk, "Writing research questions: Purpose \& examples." https://study.com/academy/lesson/writing-research-quest ions-purpose-examples.html (accessed: 14 Jun. 2019).

[2] N. H. Thuan, A. Drechsler, and P. Antunes, "Construction of design science research questions," Commun. Assoc. Inf. Syst., vol. 44, no. 1, p. 20, 2019.

[3] J. Recker, Scientific research in information systems: a beginner's guide. Springer, 2013.

[4] S. Gregor and A. R. Hevner, "Positioning and presenting design science research for maximum impact," MIS Quarterly, vol. 37, no. 2, pp. 337-355, 2013.

[5] P. Johannesson and E. Perjons, An Introduction to Design Science. Springer, 2014.

[6] R. J. Wieringa, Design science methodology: For information systems and software engineering. Springer, 2014.

[7] L. Flower and J. R. Hayes, "A cognitive process theory of writing," Coll. Compos. Commun., vol. 32, no. 4, pp. 365-387, 1981.

[8] J. A. Reither, "Writing and knowing: Toward redefining the writing process," Coll. Engl., vol. 47, no. 6, pp. 620-628, 1985.

[9] J. Wellington, "More than a matter of cognition: An exploration of affective writing problems of post-graduate students and their possible solutions," Teach. High. Educ., vol. 15, no. 2, pp. 135-150, 2010.

[10] T. Rapley, Doing conversation, discourse and document analysis. Sage, 2008.

[11] C. Bereiter and M. Scardamalia, The psychology of written composition. Lawrence Erlbaum, 1987.

[12] S. P. Turbek, T. M. Chock, K. Donahue, et al. "Scientific Writing Made Easy: A Step-by-Step Guide to Undergraduate Writing in the Biological Sciences," Bull. Ecol. Soc. Am., vol. 97, no. 4, pp. 417-426, 2016.

[13] C. W. Keys, "Revitalizing instruction in scientific genres: Connecting knowledge production with writing to learn in science," Sci. Educ., vol. 83, no. 2, pp. 115-130, 1999.
[14] J. A. Langer and A. N. Applebee, How Writing Shapes Thinking: A Study of Teaching and Learning. ERIC, 1987.

[15] A. R. Hevner, "A Three Cycle View of Design Science Research," Scandinavian Journal of Information Systems, vol. 19, no. 192, pp. 87-92, 2007.

[16] R. S. Caffarella and B. G. Barnett, "Teaching doctoral students to become scholarly writers: The importance of giving and receiving critiques," Stud. High. Educ., vol. 25, no. 1, pp. 39-52, 2000.

[17] S. V. Kotsis and K. C. Chung, "How to submit a revision and tips on being a good peer-reviewer," Plast. Reconstr. Surg., vol. 133, no. 4, p. 958, 2014.

[18] H. Cohen, "How To Overcome Blank Screen Syndrome: The Ultimate Guide." https://heidicohen.com/overcome-blank-blog-post-syndr ome/ (accessed: 14 Jun. 2019).

[19] L. L. Khaw, "A problem-solution based framework for writing the introductions of research articles in electronic and communication engineering: Pedagogical implications," in Proc. IEEE Global Eng. Educ. Conf., pp. 119-124, 2017.

[20] M. Baer, K. T. Dirks, and J. A. Nickerson, "Microfoundations of strategic problem formulation," Strateg. Manage. J., vol. 34, no. 2, pp. 197-214, 2013.

[21] E. W. Patterson, "Structuring the composition process in scientific writing," Int. J. Sci. Educ., vol. 23, no. 1, pp. 1-16, 2001.

[22] J. Morley, "Academic phrasebank." http://www. phrasebank.manchester.ac.uk/ (accessed: 14 Jun. 2019).

[23] J. Howlett, "Productivity Tip: TODO Items With Eclipse Java Code." http://www.codestore.net/store.nsf/ unid/BLOG-2012082 1-0311 (accessed: 14 Jun. 2019).

[24] G. Navarro, "A guided tour to approximate string matching," ACM Comput. Surv., vol. 33, no. 1, pp. 31-88, 2001.

[25] M. C. Tremblay, A. R. Hevner, and D. J. Berndt, The Use of Focus Groups in Design Science Research, pp. 121-143. Springer, 2010.

[26] D. L. Morgan, Focus groups as qualitative research. Sage publications, 1988.

[27] M. Engin, "Research Diary: A Tool for Scaffolding," Int. J. Qual. Methods, vol. 10, no. 3, pp. 296-306, 2017.

[28] K. Cho and C. D. Schunn, "Scaffolded writing and rewriting in the discipline: A web-based reciprocal peer review system," Comput. Educ., vol. 48, no. 3, pp. 409-426, 2007.

[29] A. Mizumoto, S. Hamatani, and Y. Imao, "Applying the bundle-move connection approach to the development of an online writing support tool for research articles," Lang. Learn., vol. 67, no. 4, pp. 885-921, 2017.

[30] L. Anthony and G. V. Lashkia, "Mover: a machine learning tool to assist in the reading and writing of technical papers," IEEE T. Prof. Commun., vol. 46, no. 3, pp. 185-193, 2003.

[31] E. Cotos, Genre-based automated writing evaluation for L2 research writing: From design to evaluation and enhancement. Springer, 2014.

[32] T. Whisperer, "Writing in the middle," 2015. https://thesiswhisperer.com/2015/06/03/writing-in-the-m iddle/ (accessed: 14 Jun. 2019). 